\title{
EL PAPEL DE LA MEMORIA EN LA RECOMPOSICIÓN DE CIUDADES DEVASTADAS.
}

\author{
Carlos ITRIAGO PELS
}

Aún siendo objetos frágiles, las ciudades tienen una capacidad intrínseca para recomponerse en caso de desastres y destrucción no programada. Este artículo incursiona en los procesos de recomposición formal de las ciudades sometidas a eventos de destrucción violenta, y en el papel de la memoria y el olvido como herramientas creativas para afrontar los proyectos de reconstrucción.

En el artículo se analizan las distintas aproximaciones proyectuales desde esta óptica, basadas en el estudio de una serie de casos de ciudades destruidas por causas de guerra y cuyos procesos de recomposición ya se han dado por culminados. Finalmente, el artículo ofrece una clasificación de estrategias que definen un universo de actuaciones y de posibilidades relacionadas con la copia, la transformación y la omisión de elementos de la ciudad previa en la nueva ciudad recompuesta.

Recomposición urbana, reconstrucción de ciudad, destrucción urbana, memoria colectiva.

\section{THE ROLE OF MEMORY IN RE-COMPOSITION OF DEVASTATED CITIES.}

Cities are fragile objects, however, they have an intrinsic ability to recompose after disaster and unscheduled destruction. This article analyzes the processes of formal re-composition of cities under violent destruction events, and the role of memory and forgetfulness as creative tools facing with reconstruction projects.

The author discusses the various approaches seen from this perspective, based on cases of study of war-torn cities that already have closed their reconstruction processes. Finally, the article offers a classification of strategies that define a universe of actions and possibilities associated with copying, transforming and omission of elements present on the previous state of the city.

Urban restructuring, Rebuilding, War-torn cities, Collective memory, Devastation, Re-composition, Resilient city, Disaster Relief, City Planning, Urban development. 
Sobre la vulnerabilidad de las ciudades, y su fragilidad se ha escrito mucho. Universidades como el MIT, Stuttgart y Durham, mantienen el tema dentro de sus líneas de investigación y no pocos autores le han dedicado miles de páginas. ${ }^{1}$ Es por ello que la investigación que respalda este artículo ${ }^{2}$ no fue afrontada como otra exploración más sobre la fragilidad y la destrucción de ciudades. Más bien se enfocó en un momento posterior y más optimista: el estudio de la acción positiva de la recomposición física y morfológica posterior a eventos de destrucción masiva y no prevista. En particular, en la estrecha relación que existe entre esos procesos y la memoria.

Por regla general, cuando una ciudad devastada se enfrenta a la recomposición es porque lo peor ya ha pasado y ha dado paso a un tiempo de esperanza. Es el momento en el cual, tanto las autoridades, los ciudadanos y los encargados de acometer los proyectos suelen enfrentarse al futuro con una mezcla de sentimientos. En primer lugar, la incertidumbre, expresada a través de dudas y temores sobre las estrategias y los resultados; sobre la gestión y las finanzas; sobre las heridas y sus remedios.... Pero también les embarga una especie de "sentido de oportunidad" que descubren detrás de la tragedia y que les da fuerzas para corregir, mejorar y modernizar la ciudad.

Paul Goldberger inicia su libro Up from Zero diciendo que no existe un manual ni una hoja de ruta que le explique a una ciudad que hacer cuando sus edificios desaparecen de pronto y sólo queda un vacío en su corazón. "De hecho, es lo que quisiéramos tener..."3

Recordamos el sentimiento de pérdida e incertidumbre que inundaba al mundo en aquellos días posteriores al derribo de las torres gemelas y comprendemos el ánimo con que Goldberger escribió esas líneas. Sin embargo, ese manual con el que sueña Goldberger no es posible dada la naturaleza

1 Una bibliografía muy condensada puede incluir a los siguientes textos: BECHIR, Kenzari (ed), Architecture and Violence,Barcelona: Actar, 2011; VIRILIO, Paul, Ville panique. Ailleurs commence ici, París: Galilée, 2004; NOSSACK, Hans Erich. The end. Hamburg 1943, Connecticut: The University of Chicago Press, 2004; Lawrence J. Vale; The Resilient City: How Modern Cities Recover from Disaster. USA: Oxford University Press, 2005. DURTH, WERNER y GUTSCHOW, NIELS; Träume in Trümmern. Munich: Dtv wissenschaft, 1993. SCHNEIDER, Jane \& SUSSER, Ida (editores); Wounded cities. Destruction and reconstruction in a globalized world. Oxford: Berg, 2003. FERNÁNDEZ-ALBA, Antonio y LÓPEZ ALBALADEJO, J; WTC 11.9.01. Murcia: COAATR, , 2003. GRAHAM, Stephen; Cities, War, and Terrorism: Towards an Urban Geopolitics. Boston: Blackwell Publishing Professional , 2004. AUGUÉ, Marc; Le temps en ruines. París: Galilée, 2003. ASTOLFI, Astrid; Reconstruction après la Guerre; Paris: L'Harmattan, 1999. PLUM, Giles; L'architecture de la reconstruction. Mayenne: Nicolas Chaudun. 2011.

2 Investigación elaborada por el autor en el Programa de doctorado de urbanismo de la Universidad Politécnica de Cataluña. Ver Itriago Pels, Carlos Teodoro; Sobre copias, transformaciones y omisiones. La recomposición de ciudades devastadas en http://www.tdx.cat/handle/10803/6963. Última visita 2012-04-25.

3 Goldberger, Paul; Up from Zero. Nueva York: Random House, 2005, p.-xi 
propia de las operaciones de recomposición de ciudades devastadas. Sin embargo, nuestra disciplina sí cuenta con los instrumentos para actuar en un campo del que no se ha librado ninguna civilización. Abordamos esta investigación con el objetivo de comprender las posibles formas de actuación ante estos eventos.

¿Cómo son los procesos de recomposición y sus herramientas?

¿Por qué se decide recomponer de una manera y no de otra?

¿Qué pistas podemos obtener del pasado para actuaciones en el futuro?

Nuestra investigación se justificó en la capacidad real que tienen las ciudades de recomponer sus estructuras por medio de proyectos específicos para esos fines y fue planteada como una plataforma para estudiar la regeneración de los tejidos urbanos en vacíos creados de forma violenta y no programada, sobre sectores de intensa actividad urbana.

Para ello se sistematizó de manera crítica y comparativa la información de un grupo representativo de proyectos urbanísticos de recomposición para poder estudiar sus instrumentos, metodologías y estrategias, y su estrecha relación con la memoria, entendida como una herramienta creativa.

De hecho queríamos demostrar que estas operaciones, en general, han servido como laboratorio para germinar nuevos instrumentos, procesos e ideas ligados al papel de la memoria como herramienta.

Por otra parte, la investigación también debía ilustrar la capacidad real de cambio de una ciudad enfrentada a las contradicciones entre el estado de emergencia y los requerimientos de la ciudad en el largo plazo.

La necesidad de centrar la investigación para un análisis riguroso nos llevó a limitar la muestra de los casos a un número reducido que cumpliera una serie de condiciones que fuimos aislando mientras se desarrollaban los trabajos. Los proyectos de recomposición seleccionados eran casos paradigmáticos y representativos que cumplían con cuatro condiciones: En primer lugar, eran casos donde la destrucción se concentró sobre sus cascos centrales como producto de actos de guerra, dadas las características propias de este tipo de destrucción. Limitamos la selección al período de la segunda guerra mundial, debido a la intensidad del debate disciplinar de las décadas anteriores en Europa y a las características propias de la destrucción, que alcanzó una extensión sin precedentes. Pero también por la perspectiva histórica que nos brindaban los 60 ańos que nos separan del momento en que se dieron por cerradas muchas operaciones. Por último, la selección se centró en los casos más emblemáticos de este período en Alemania, Francia, Holanda, Inglaterra, Italia y Polonia, asegurando un universo de casos dentro de realidades 


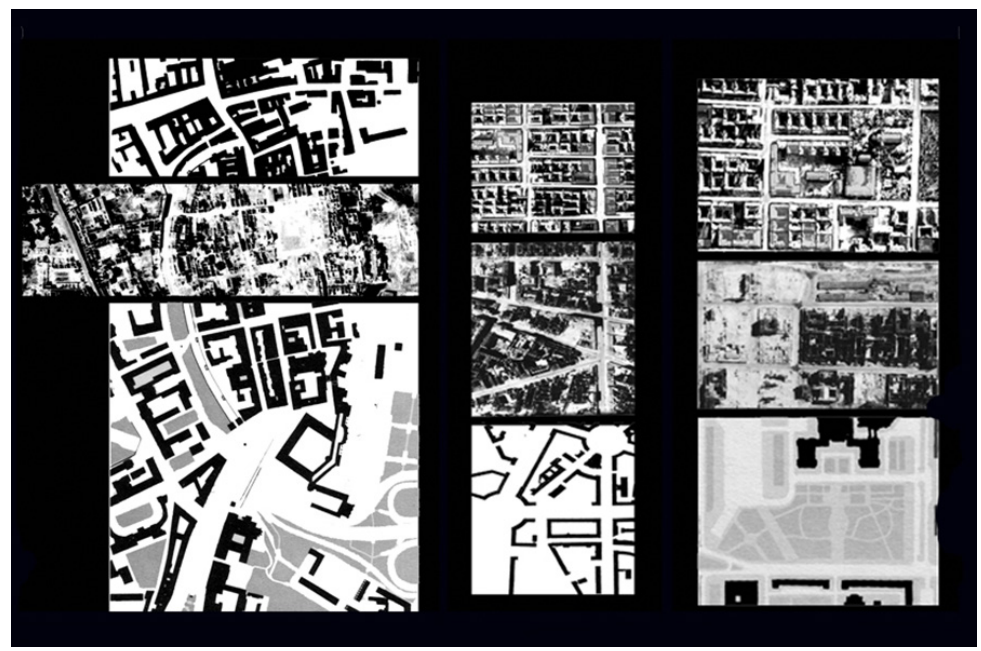

Figura 1. Este tríptico muestra a los tres racimos de estrategias aplicados en una única ciudad, Varsovia, a poca distancia unas de otras y en muy poco tiempo. En las columnas se muestran los fragmentos de ciudad, y en las filas horizontales El Estado Previo, el Estado de separación y el Estado Liminal de cada uno. En la columna de la Izquierda: Stare Miasto. Uno de los ejemplos emblemáticos de estrategias autorreferentes. En la columna del centro la Avenida Marszalkowska, modelo de estrategias emancipadoras y en la columna de la derecha la operación para implantar el Palacio de las ciencias y la cultura sobre el espacio del antiguo Gueto de Varsovia. Fuente: elaboración propia.

nacionales europeas distintas que permitieron comprender sus diferencias y similitudes.

Siguiendo estos criterios nos aseguramos una variedad que brindaba un campo propicio para adentrarnos en discusiones tanto de tipo instrumental como de orden estilístico, que intuíamos eran importantes en estas operaciones. A partir de ellos escogimos un total de $12 \operatorname{casos}^{4}$ que se analizaron comparativamente tanto en sus estrategias como en las características propias de las transformaciones morfológicas, es decir, estudiando aquello que se copió, se transformó o se omitió en cada caso siempre teniendo el papel de la memoria como leit-motiv.

Para poder comparar sistemáticamente los distintos casos seleccionados y la capacidad transformadora de las operaciones, necesitábamos un marco de referencia común que de alguna manera estableciera las pautas de la comparación. El modelo de los "Fenómenos de Paso" de Charles-Arnold Van Gennep ${ }^{5}$ nos ofreció este marco, dentro de una estructura repetitiva y confiable.

Según este autor, toda sociedad o individuo que sufre un cambio de estado, lo hace según una estructura invariable de cinco pasos continuos y conse-

4 Estos fueron los casos analizados con mayor profundidad: Varsovia, Dresde, Stalinallee en Berlín, Münster, Hamburgo, Rotterdam, Le Havre, Saint-Malo, Amiens, Paternoster y Barbican en Londres, y Florencia.

5 VAN GENNEP, Arnold (1909), Los ritos de paso, Madrid: Taurus 1986 
cutivos: El Estado Previo; el Estado de Separación; el Estado Liminal; el Estado de Reincorporación y el Estado Actual. Hemos podido comprobar que los procesos de Destrucción/Recomposición responden a esta estructura fija y confiable que parte de un estado previo y que pasa por un evento que puede remover de ese estado al individuo, llevándolo a una nueva condición, a la que denominamos "estado de separación."

El estado que Van Gennep denomina "liminal," es para nuestro análisis el período de las discusiones disciplinares y de los proyectos. Por último, el modelo reconoce un estado de "reincorporación," en el cual, la ciudad repuesta, adopta su nuevo rol.

Alrededor de estos estados ha girado la investigación, ya que, si de recordar u olvidar se trata, la comparación crítica entre el estado previo de la ciudad y el proyecto de recomposición permitirá definir las estrategias de actuación en las que se enmarcan estas operaciones.

Los primeros resultados de ese análisis comparativo nos llevaron a proponer el uso de la palabra "recomposición" en sustitución de "reconstrucción", ya que hemos constatado que estas operaciones se mueven en un campo especulativo en medio de complejos trabajos de "composición", que no pretenden en ningún caso volver a construir las ciudades devastadas de manera similar a su estado previo, como sugiere la palabra "reconstrucción".

Iniciamos la investigación con la convicción de que, aunque la destrucción violenta y no programada de una ciudad puede dejarla en una virtual tabla rasa, siempre subsiste su pasado, aunque sólo sea en forma de recuerdos. De hecho, el pasado siempre permanece intacto en las ciudades... Sin embargo, aún cuando la ciudad destruida no estuviera dispuesta a seguir viviendo en el pasado, no puede impedir que éste condicione de cierta forma su futuro.

Este pasado se expresará a través de un único objeto: la memoria. Ésta es la única herramienta que tenemos para traer de vuelta el pasado al presente.

Ahora bien, si como afirma Ricoeur, ${ }^{6}$ las sociedades tienen la obligación para con ellas mismas de transmitir transgeneracionalmente sus aciertos y valores, entonces, a la hora de afrontar la recomposición, se enfrentarán a la disyuntiva entre recordar u olvidar. La decisión que tomen, definirá su destino y el de su herencia urbana.

Dentro de ese marco se han respaldado muchas iniciativas para restituir un pasado cuidadosamente seleccionado; pero en otros tantos se ha justificado establecer una narración totalmente novedosa para la ciudad, alejándose de lo "ya visto". 
Pensemos en una ciudad que vive sin alteraciones devastadoras. En esas condiciones de estabilidad, su inercia actúa como una narración continua que le permite permanecer siendo "ella misma" en el tiempo.

Sin embargo, cuando se enfrenta a un evento de destrucción masiva, la narración se interrumpe y para retomarla habrá que hacer uso de la memoria. Dependiendo de lo prolongado y profundo de la ruptura, el silencio que genera la destrucción puede inducir al olvido y por consiguiente suscitar una nueva narración, o por el contrario, puede promover que se retome el hilo de la narración interrumpida, haciendo prevalecer recuerdos cuidadosamente seleccionados del pasado.

No obstante, como ha dicho tantas veces Umberto Eco, la función de la memoria no es sólo recordar, sino filtrar. De allí que la memoria nunca producirá una estampa perfecta del objeto recordado, porque su función, es producir una imagen simplificada y reinterpretada desde el aquí y el ahora. El uso selectivo e intencionado de la memoria, recordando u olvidando, será la clave para definir las estrategias proyectuales con las cuales afrontar la recomposición.

A través de un análisis detallado de los casos seleccionados, hemos propuesto una clasificación dividida en tres racimos de estrategias definidas por la actitud para con la memoria de la ciudad, expresada en aquello que se transforma, se omite o se copia, y las formas como lo hace. Estas estrategias son las estrategias autorreferentes; las estrategias refundadoras y las estrategias emancipadoras.

Estos racimos de estrategias no son excluyentes entre sí. Muy por el contrario pueden convivir con más o menos armonía simultáneamente en fragmentos distintos de una ciudad devastada, siendo así que los elementos que con más insistencia son objeto de copia o transformación en operaciones de recomposición, lo son así, en todas las estrategias. Por tanto, las diferencias que definirán unas estrategias de otras, estribarán en la intensidad con que se le dé preferencia a la copia, a la transformación o a la omisión de ciertos elementos.

Como ejemplo de la convivencia de las estrategias en una misma ciudad proponemos el caso de Varsovia después de la segunda guerra mundial. Esta operación se llevó a cabo en distintos fragmentos de la ciudad respondiendo a ideologías, necesidades e incluso realidades contrapuestas. Los responsables de aquellos proyectos desplegaron tres aproximaciones estratégicas opuestas, que encajan perfectamente con nuestra clasificación. Por una parte, una recomposición refundadora de un amplísimo sector de la ciudad que terminó de borrar del mapa lo poco que quedaba en pie del tristemente célebre Gueto. Por otra parte, una operación del tipo emancipadora, que de forma pragmática, se apropia de piezas, estructuras y argumentos de la ciudad destruida 
para elaborar un nuevo discurso apropiado a la nueva realidad socialista del país. Y, por último, la recomposición autorreferente del casco antiguo de la ciudad estrechamente ligado a la memoria colectiva, operación celebrada como la más fiel recomposición del pasado de la segunda postguerra europea.

Ahora bien, si las ansias modernizadoras son una característica típica de cualquier operación urbana, parece evidente que las operaciones de recomposición no deberían escapar de esa aspiración. Entonces ¿por qué encontramos tantos casos, como el del casco central de Varsovia, que copian con exactitud aparente sus estructuras destruidas? Al actuar así, ¿acaso se renuncia a la oportunidad que le brindaba la destrucción para modernizarse?

En realidad no lo hacen, sino que actúan de forma menos evidente en ese sentido. Efectivamente, la búsqueda de referencias propias, dentro de los registros del pasado más o menos reciente de la ciudad destruida privilegiando una "imagen previa", cuidadosamente seleccionada, es el sello que caracteriza a las estrategias que denominamos autorreferentes.

En primer lugar, estas estrategias reconocen los valores urbanísticos particulares del estado previo de la ciudad destruida, y se acogen a ellos con confianza. No obstante, el reconocimiento de esos valores no exige su reproducción con exactitud milimétrica, sino una recreación de un cuadro armonioso que vincule la nueva imagen con el pasado. Incluso en Varsovia, donde se creó una escuela de artesanías de la construcción para poder reproducir con exactitud la pátina del tiempo, la copia no pretendió ser idéntica al original.

Adicionalmente, detrás de ciertos lenguajes estéticos y formales exteriores tomados del pasado reciente, siempre encontraremos diluidos importantes avances de la modernidad que actualizan esa imagen y dan viabilidad a la operación. Por último, el establecimiento de estas estrategias está enmarcado en un proyecto social con una marcada ideología que busca reafirmar la identidad herida de la ciudad cumpliendo el deber de retransmitirlas a las generaciones futuras.

Lejos de buscar la semejanza perfecta con algún estado previo, pensamos que las estrategias autorreferentes son una aplicación práctica contemporánea del pensamiento de Viollet-le-Duc. Según una sentencia del famoso arquitecto francés, restaurar es restablecer a un estado que pudo no haber existido jamás en esa forma dada. Ese estado, no obstante, emula lo que alguna vez existió, sustentado en ciertas formas de nostalgia.

En el extremo opuesto de nuestra clasificación están las estrategias refundadoras, cuyo espíritu es el de la renovación total de las estructuras de la ciudad, partiendo de la premisa de que con la devastación se ha roto la continuidad con el pasado. Esto brinda la oportunidad de ir en busca de una ciudad ajena a la anterior. Estas estrategias se reconocen por aprovechar la oportunidad 
que brinda la destrucción para llevar a cabo operaciones que en otras circunstancias hubieran sido utópicas. De tal manera que el sentido de la oportunidad tiene una respuesta contundente y un marcado carácter experimental, que las aleja de cualquier consideración hacia el pasado. Lo "no visto aún", que escapa de la añoranza, tiene un peso particular en unas estrategias que nunca apuestan por modelos conocidos.

Son operaciones cargadas de contenidos ideológicos trascendentales para la operación; por lo mismo, sus promotores no dudan en imponerlas en la ciudad. A nuestro juicio, las estrategias refundadoras de la segunda posguerra fueron auténticos laboratorios de urbanismo en los que se prepararon muchos de los caminos del urbanismo contemporáneo. ${ }^{7}$

Sin embargo, al profundizar en los casos de estudio, detectamos que las operaciones que ponen en marcha estrategias refundadoras suelen verse en la necesidad de incorporar referencias de la ciudad destruida; de allí que afirmáramos anteriormente que las estrategias no son excluyentes entre ellas.

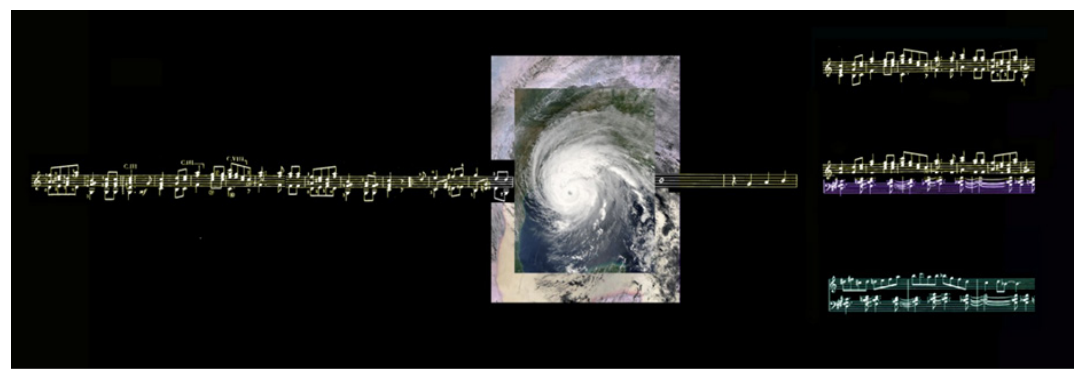

Figura 2. La melodía de la izquierda expresa la narración continua de una ciudad que le permite permanecer siendo "ella misma" en el tiempo; pero cuando se enfrenta a un evento de destrucción masivo (en el centro de la imagen), la narración se interrumpe y para retomarla habrá que hacer uso de la Memoria. Esta situación puede suscitar una nueva narración, o por el contrario, puede promover que se retome el hilo interrumpido con recuerdos cuidadosamente seleccionados del pasado. Este esquema es la base de las estrategias propuestas en nuestra investigación. Fuente: elaboración propia.

Pongamos como ejemplo el proyecto de recomposición del puerto de Le Havre en Francia. Cuando el "Atelier de Reconstrucción de Le Havre Auguste Perret" se enfrentó a la realidad del proyecto de recomposición más emblemático de Francia, ideado como ejemplo de la más pura modernidad (más no de los ideales del movimiento moderno) se vieron en la necesidad de renunciar a propuestas realmente agresivas como la "coquille en beton" (con la que pretendían elevar el nivel de las calles para crear un gran sótano continuo destinado a los servicios comunes de la ciudad) y simultáneamente accedieron a las presiones que les exigían respetar la secuencia espacial de

7 Muchos autores no dudan en enlazar estrategias ligadas al proyecto urbano actual con aquellas experimentadas durante los procesos de recomposición de la segunda posguerra. La exploración de estos vínculos puede abrir nuevas líneas de investigación. Véase, como muestra, a Sola Morales (de), Manuel; "Another modern tradition. From the break of 1930 to the modern urban project" en Lotus International, № 64. (En castellano en UR, n. 5, Barcelona 1987. 
calles y plazas para salvaguardar la identidad del viejo Le Havre. Cumpliendo con esta exigencia, Auguste Perret hacía una reivindicación del plano de expansión de Le Havre de 1838 en un proyecto en el cual privaron en todo momento estrategias de tipo refundador.

Lo contrario también es común. Muchas de las operaciones que ponen en marcha estrategias de tipo autorreferente, conceden espacio a la modernización más allá de lo que se podría esperar, como es el caso de la ciudad amurallada de Saint Malo.

Las estrategias puestas en marcha allí, consistieron, primero, en la reparación minuciosa, casi milimétrica de las cuatro fachadas de la ciudad, es decir, de las murallas exteriores. De esta manera, la ciudad respetaba su estampa, reconocible a distancia como una especie de objeto monolítico. Sin embargo, en la ciudad intramuros, simplemente se elaboraron unas normas para obtener un resultado armonioso. Estas normas permitieron, incluso, la demolición de cientos de edificios rescatables que habían sido catalogados al comenzar los trabajos. Según la norma, los nuevos edificios sólo debían mantener un lenguaje simplificado de la arquitectura del norte de Bretaña.

Los dos ejemplos anteriores se repiten de una manera u otra en prácticamente todos los casos estudiados pertenecientes a una $\mathrm{u}$ otra de las estrategias descritas, siendo así que con frecuencia las estrategias se entremezclan de tal manera que les aleja de los extremos del espectro. Por ello, nos hemos visto movidos a definir el tercer racimo de estrategias, al que denominamos estrategias emancipadoras.

Estas estrategias son el fruto de una interpretación pragmática de la relación de la herencia recibida con la modernidad deseada. Esto las diferencia de los

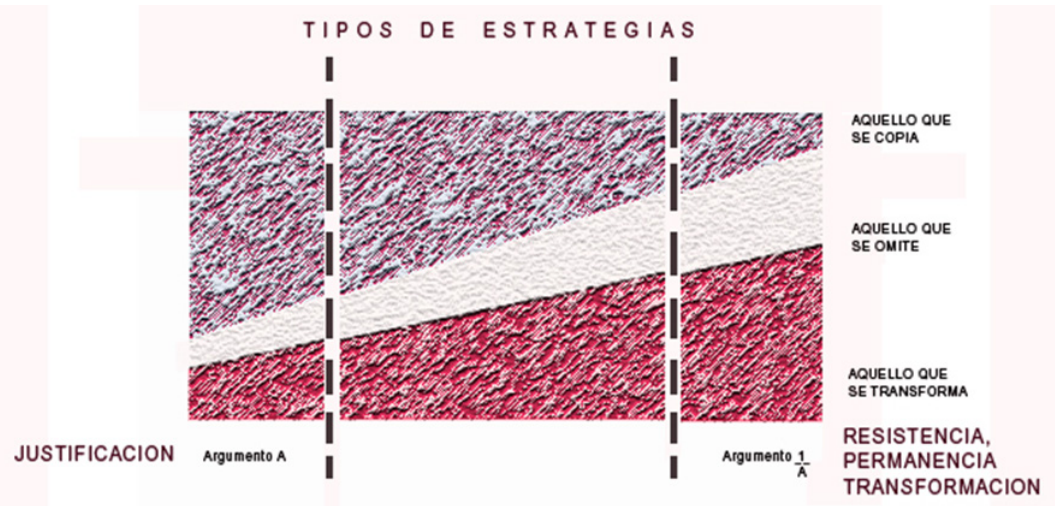

Figura 3. Los elementos que son objeto de copia, transformación u omisión, lo son sea cual sea la estrategia que se aplique. Entonces, la diferencias estribarán en la intensidad con que se le dé prioridad a la copia 0 a la transformación de ciertos elementos. Fuente: elaboración propia. 
otros dos racimos cargados ideológicamente. Su búsqueda se concentra en la transformación moderada de la ciudad, emancipándola de lastres que le impedían desarrollarse antes de la destrucción.

Una vez identificados los racimos de estrategias que abarcan las operaciones de recomposición de ciudades devastadas debíamos poner en evidencia las relaciones entre el pasado de la ciudad y su recomposición. Nos preguntamos entonces qué preguntas debíamos hacerles a los proyectos sobre su manera de usar la memoria como herramienta creativa.

Para responder lo anterior debemos convenir en que una ciudad recompuesta difiere de la ciudad en su estado previo en cuanto es, en sí misma, un objeto individual. Este argumento está basado en un concepto aristotélico que ilustró Magritte con la famosa "pipa que no es una pipa". Si aceptamos como cierta esa dicotomía entre ser copia de algo y, a la vez, ser un objeto en sí mismo, entonces podemos afirmar que aquello que una nueva ciudad recompuesta, aunque sea aparentemente idéntica a un original, omite o altera de su modelo, es sobresaliente e instructivo, en un modo tan crítico como lo que incluye, ya sea a través de la variante o de la copia directa. ${ }^{8}$

La aplicación de esta idea nos ayuda, por tanto, a definir las preguntas que debemos hacer a cada ciudad recompuesta:

¿Qué se copia?

¿Qué se transforma?

¿Qué se omite?

Sometiendo a ese interrogatorio a los doce casos seleccionados, se aislaron unos denominadores comunes a la hora de copiar y de transformar elementos de una ciudad destruida y recompuesta.

En primer lugar se puso en evidencia que aquello que se copia del estado previo en una ciudad recompuesta pertenece sobre todo al ámbito de las percepciones, y por tanto al universo estético de la ciudad, es decir a aquello que con mayor fuerza puede impresionar a la memoria colectiva de una sociedad. Por el contrario, se transforman los elementos que son capaces de innovar en la forma de vivir.

Cuando hablamos de copias en la trama de la ciudad devastada, nos referimos a la reproducción más o menos fiel de aquello que fue y que ya no existe porque fue destruido en la catástrofe. De lo que se trata en estos casos es de la reproducción de un objeto original que ha desaparecido físicamente para siempre. La reproducción de piezas destruidas, por medio de nuevas

8 Esta hipótesis está inspirada en una frase de George Steiner refiriéndose a la literatura. STEINER, G., Presencias reales, Barcelona: Destino, 1991, p.25 
construcciones o estructuras que busquen restablecerlas, suele conllevar un proceso de ajuste para que su comportamiento sea compatible con su nuevo estado. Así, el nuevo objeto o estructura tendrá un comportamiento que le será propio y posiblemente diferente a aquél que sustituye. Sin embargo, la relación que se establecerá con el original, en cuanto que representado por la copia, cargará de significados la existencia del nuevo objeto.

Por tanto, la copia es un recurso que se aplica sobre fragmentos de la ciudad, que luego interactúan con su entorno, generando una nueva vida que rememora la que fue destruida sin necesidad de ser totalmente idéntica. Para implementar estas operaciones los proyectistas recurren a la creación de marcos formales estrictos dentro de los cuales desarrollar los instrumentos de actuación; recurren igualmente al manejo riguroso de la documentación del pasado, y a la adecuación a las necesidades modernas por medio de operaciones paralelas y códigos de construcción adecuados a ese fin.

En nuestra investigación aislamos tres elementos que suelen ser objetos de copia: la especialidad de la ciudad; los elementos icónicos con capacidad documental, que puede incluir tanto monumentos como piezas de arquitectura menor que devienen en nuevos íconos de la ciudad; y la estampa de la ciudad.

Con este breve listado podemos afirmar, reforzando lo dicho más arriba, que la acción de copiar actúa sobre los elementos que albergan la memoria visual y espacial de la ciudad.

Por el contrario, para reconocer los elementos que suelen ser objetos de transformación debemos recordar nuevamente el sentido de la oportunidad que suele acompañar a este tipo de operaciones. La oportunidad detrás de la destrucción reside en poder aplicar correctivos sobre la desincronización entre el estado actual y las necesidades reales de la ciudad. Estos eventos permiten poner en práctica ejercicios de actualización de las estructuras urbanas, que se evidenciarán en transformaciones profundas sobre los elementos de la ciudad potencialmente modernizadores, tales como: las lógicas que se expresan en la partición del suelo; las lógicas que dan forma y sentido a la urbanización y a las infraestructuras, y las lógicas de la edificación.

El espacio en estas páginas no es suficiente para ampliar en detalle estos elementos que sí se pueden revisar en profundidad en la obra que documenta la investigación. ${ }^{9}$

Las numerosas tragedias que han afectado a tantas ciudades en el último lustro nos exige repensar el tema a la luz de otros eventos de destrucción, diferentes a la guerra convencional, para actualizar así nuestras conclusiones desde la perspectiva de la destrucción por terrorismo, guerrillas urbanas, 
revueltas civiles, desastres industriales, terremotos, inundaciones, aludes torrenciales, tsunamis, etc. Como dijimos al principio, por razones metodológicas la investigación se acotó a casos de destrucción por guerra en el ámbito europeo de la segunda postguerra, pero además se concretó a la dimensión formal y geométrica de la ciudad. Sin embargo, ¿qué pasa con las otras dimensiones de la reconstrucción? ¿Acaso los urbanistas somos ajenos a estas? La respuesta es un categórico no.

Campos como la mitigación de daños, la reconstrucción de la identidad, la gestión de la emergencia inmediata, la gestión de las infraestructuras, la priorización de las acciones, la organización del catastro, la gestión de los escombros..., todos son campos abiertos a la investigación con mucho potencial académico.

Algunos casos actuales, como la reconstrucción de Port-au-Prince en Haití, de New Orleans en Estados Unidos, del Litoral chileno después del tsunami o de Fukushima con su componente nuclear adicional, nos llevan a promover la investigación desde nuestra disciplina en un campo que siempre tendrá abierto algún frente donde actuar. ¿O preferimos quedarnos como el profeta Jeremías...lamentando la destrucción de Jerusalén? 